\title{
Lithium incorporation into a silica thin film: Scanning tunneling microscopy and density functional theory
}

\author{
Jan Frederik Jerratsch, ${ }^{1}$ Niklas Nilius, ${ }^{1, *}$ Hans-Joachim Freund, ${ }^{1}$ Umberto Martinez, ${ }^{2}$ Livia Giordano, ${ }^{2}$ and \\ Gianfranco Pacchioni ${ }^{2}$ \\ ${ }^{1}$ Department of Chemical Physics, Fritz-Haber Institut der Max-Planck Gesellschaft, Faradayweg 4-6, D-14195 Berlin, Germany \\ ${ }^{2}$ Dipartimento di Scienza dei Materiali, Università di Milano-Bicocca, via Cozzi 53, 20125 Milano, Italy \\ (Received 6 August 2009; revised manuscript received 4 November 2009; published 16 December 2009)
}

\begin{abstract}
The interaction of Li with a thin silica film grown on $\mathrm{Mo}(112)$ has been studied with low-temperature scanning tunneling microscopy (STM) and density functional theory (DFT). Thanks to the porous silica structure, single Li atoms are able to penetrate the oxide layer and bind at the metal-oxide interface. From the distinct topographic signature of the embedded atoms, the interfacial adsorption sites are determined to be along the furrows of the Mo(112) surface. With increasing Li coverage, the formation of an ordered superstructure is observed, where stripes of high and low Li density alternate in the direction perpendicular to the Mo furrows. The ordering effect is explained by the anisotropic screening response of the Mo(112) surface. The $\mathrm{Li}$ becomes cationic upon adsorption at the interface and substantially lowers the work function of the silica/Mo system. This work-function decrease is directly deduced from the downshift of the silica conduction band, as determined with STM conductance spectroscopy and DFT calculations. The derived interrelation between work function and Li coverage strongly deviates from the universal behavior found on metal surfaces, reflecting the superior screening of the $\mathrm{Li}^{+}$ions in their binding sites at the metal-oxide interface.
\end{abstract}

DOI: $10.1103 /$ PhysRevB.80.245423

PACS number(s): 73.30.+y, 68.43.Fg, 73.61.Ng, 68.37.Ef

\section{INTRODUCTION}

The alkali metals stick out from all other elements by their exceptionally low ionization potential. This peculiarity reflects the weak bonding of their unpaired $s$ electron that is easily removed from the valence shell. Partial ionization of the alkali therefore governs the adsorption on metal substrates. Whereas on noble metals, almost a complete electron charge is transferred into the support, the alkali-surface interaction has more covalent character on transition metals and semiconductors. ${ }^{1,2}$ On molybdenum and tungsten, for example, hybridization between the valence $s$ state of the alkali and the $d$ band of the metal dominates the interaction, resulting in a strong polarization of the alkali $s$ electron toward the support. ${ }^{3}$ In any case, the charged/polarized adatom gives rise to a positive surface dipole that triggers a considerable work-function decrease in the system. At higher alkali load, these surface dipoles experience a repulsive interaction, which gradually inhibits the electron transfer away from the alkalis. As a result, depolarization of the adlayer takes place and the alkalis become metallic again, causing the work function to increase toward the bulk value of the respective admetal. The typical work function versus coverage curve for alkali systems is therefore characterized by a sharp decrease, reflecting initial ionization of the adatoms, followed by a slow increase due to the gradual metallization of the adlayer at higher load. ${ }^{1,2,4}$ At intermediate coverage, the alkali atoms often self-assemble into regular arrays on the metal surface in order to maximize their mutual distance. ${ }^{5-7}$ By this means, the repulsive dipole-dipole interaction and the subsequent layer depolarization are restrained. Already this simple picture, first suggested by Langmuir and Gurney, ${ }^{8}$ provides a good description for the general adsorption behavior of alkali atoms on metal surfaces.

The small ionization potential of the alkalis is of large technological importance, for instance, to produce low work- function electrode materials with high electron emissivity. Furthermore, alkali atoms are used as promoters in catalytic applications, as they are able to supply weakly bound electrons for reduction processes..$^{1,9,10}$ The main technological challenge in the use of alkalis is their protection from unwanted chemical reactions that would cause rapid degradation of their desired properties. For this purpose, the alkali atoms are usually embedded in metal or oxide matrices, which inhibit the contact to a reactive environment but also suppress the intended effect on the work function. In a recent theoretical work, an elegant way has been proposed to combine the two requirements. ${ }^{11}$ The alkali atoms are hereby placed at the interface between an ultrathin $\mathrm{SiO}_{2}$ film and a Mo(112) single crystal. In their interfacial binding positions, the alkali are well protected by the inert oxide layer but still close enough to the surface to lower the work function of the system. Furthermore, the adsorption sites at the metal-oxide interface are accessible even after film preparation because the porous nature of silica enables the smaller alkali atoms, $\mathrm{Li}$ and $\mathrm{Na}$, to diffuse through the oxide surface. ${ }^{11}$ The possibility of $\mathrm{Li}$ incorporation into silica/Mo(112) has already been demonstrated by comparing the Au adsorption behavior of the clean and Li-doped system. ${ }^{12}$ While the pristine film does not interact with $\mathrm{Au}$ atoms except for defects, the modified silica is able to stabilize Au atoms and clusters even on regular surface sites. The underlying binding mechanism was traced back to a Li-mediated electron transfer into the gold, which enables efficient electrostatic and polaronic interaction schemes with the oxide surface. ${ }^{13}$

In this work, we provide more details on the Li interaction with the silica/Mo system, employing low-temperature scanning tunneling microscopy (STM) and density functional theory (DFT) as investigative tools. In particular, we want to shed light on the influence of the oxide film on the $\mathrm{Li}$ adsorption behavior and the resulting work-function evolution. 

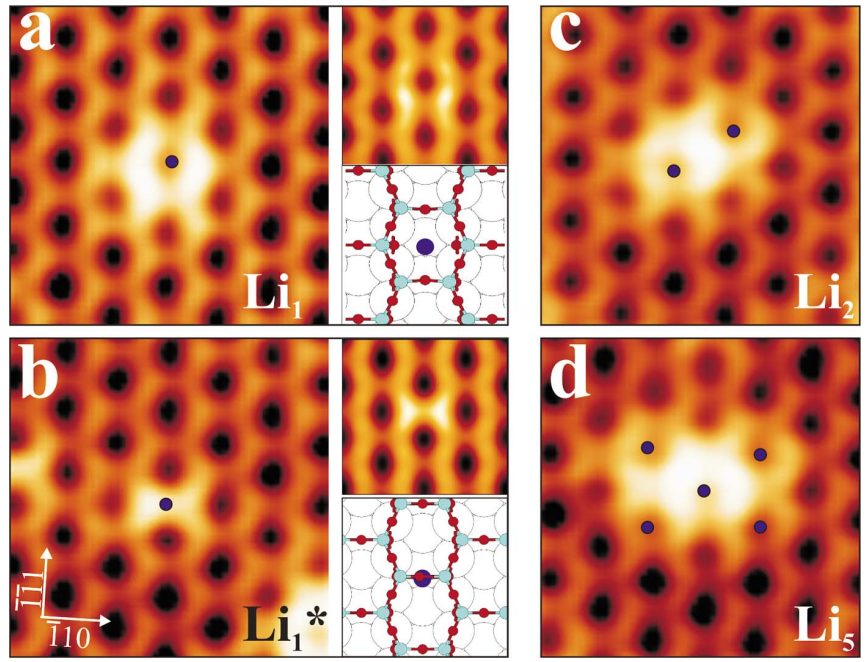

FIG. 1. (Color online) [(a) and (b)] Experimental (0.1 V, 3 $\left.\times 3 \mathrm{~nm}^{2}\right)$ and simulated STM images $\left(0.5 \mathrm{~V}, 1.8 \times 1.6 \mathrm{~nm}^{2}\right)$ as well as structure model of a single Li atom bound to two different interface sites of silica/Mo(112). The oxygen and silicon atoms in the scheme are marked by small red and large cyan spheres, respectively, whereas the $\mathrm{Li}$ is shown in dark blue. [(c) and (d)] STM images of two larger Li aggregates $\left(0.1 \mathrm{~V}, 3 \times 3 \mathrm{~nm}^{2}\right)$. The position of the interfacial $\mathrm{Li}$ atoms is marked by dots.

Furthermore, the spatial distribution of Li adsorbates at the metal-oxide interface is analyzed and explained in terms of the distinct screening response of the Mo(112) surface.

\section{EXPERIMENT AND THEORY}

All measurements have been performed with a custommade ultrahigh-vacuum STM operating at $10 \mathrm{~K}$. Topographic and electronic sample information is obtained with constantcurrent imaging and differential conductance $(d I / d V)$ spectroscopy using lock-in technique. The preparation of the silica film follows the recipe given in Ref. 14. A sputtered/ annealed $\mathrm{Mo}(112)$ surface, consisting of a [ī11]-oriented row and furrow structure, is used as support (Fig. 1). Prior to film preparation, the surface is predosed with $50 \mathrm{~L} \mathrm{O}_{2}$ and then exposed to a monolayer of $\mathrm{Si}$ in an $\mathrm{O}_{2}$ ambiance of 1 $\times 10^{-7}$ mbar at $550 \mathrm{~K}$. Subsequent annealing to $1200 \mathrm{~K}$ results in the formation of a crystalline oxide film, comprising a single layer of corner-sharing $\mathrm{SiO}_{4}$ tetrahedrons. ${ }^{14,15}$ Three of the $\mathrm{O}$ atoms hereby form bonds to neighboring $\mathrm{Si}$ atoms in the plane while the fourth one sits below the $\mathrm{Si}$ and anchors the film to the support. The in-plane -Si-O- units are arranged in six-membered rings of $5 \AA$ diameter, which enclose a hole reaching down to the Mo surface (Fig. 1). The $\mathrm{Si}$ and $\mathrm{O}$ surface atoms are formally in the +4 and -2 oxidation states, respectively, rendering the silica surface chemically inert against molecular adsorption. ${ }^{16}$ The most prominent structural defects are antiphase domain boundaries between neighboring oxide patches. Along those boundary lines, the regular six-membered -Si-O- rings are replaced by eightmembered entities having a larger central opening. ${ }^{17} \mathrm{The} \mathrm{Li}$ atoms are deposited from a commercial dispenser onto the freshly prepared surface at $77 \mathrm{~K}$.
DFT calculations are performed with the generalized gradient approximation as implemented in the VASP code, using the PW91 exchange-correlation functional and a plane-wave basis set (energy cutoff of $400 \mathrm{eV}$ ). ${ }^{18,19}$ The electron-ion interaction is described by the projector augmented wave method. ${ }^{20}$ A $(4 \times 2)$ supercell with a composition of $\mathrm{Mo}_{56} \mathrm{Si}_{8} \mathrm{O}_{20}$ is used to model the $\mathrm{Li}$ interaction with the oxide film. The cell can hold one to eight Li atoms, corresponding to a coverage $\Theta$ of $0.25-2$ atoms per silica ring. In selected cases, also larger $(6 \times 4)$ cells are used to simulate the spatial distribution of the $\mathrm{Li}$ atoms and to generate STM images on the basis of the Tersoff-Hamann approach. ${ }^{21}$ The slabs are always separated by at least $10 \AA$ of vacuum and a dipole correction scheme is applied. The work function $\phi$ of the metal/oxide system has been defined as the energy of the vacuum level, determined as the self-consistent potential in the vacuum with respect to the Fermi energy of the metal.

\section{RESULTS AND DISCUSSION}

\section{A. Single $\mathrm{Li}$ adsorbates}

Already the deposition of small Li amounts onto the silica film (below 0.05 atoms per silica pore) leads to the appearance of distinct adsorption structures in the STM. Two configurations can be distinguished, namely, ringlike structures that coincide with one -Si-O- hexagon [Fig. 1(a)] and $\mathrm{X}$-shaped elements oriented along the $\mathrm{Mo}[\overline{1} 10]$ direction [Fig. 1(b)]. Larger adstructures are always assembled from those elementary configurations, whereby particularly high imaging contrast is observed at the intersection points of two adjacent elements. For example, the structure in Fig. 1(c) is assigned to a Li dimer while the pattern in Fig. 1(d) might be assembled from five elements positioned on neighboring -Si-O- rings. In all cases, not the adatom itself but distinct building blocks of the silica film seem to be responsible for the topographic contrast. A similar behavior was earlier observed for Pd and Ag atoms and explained by the fact that the atoms actually occupy binding sites below the surface at the metal-oxide interface. ${ }^{22,23}$ The distinct contrast is hereby triggered by a hybridization of the valence $s$ state of the adatom and the $\mathrm{O} 2 p$ orbitals of the surface oxygen atoms, which locally increases the unoccupied state density of the silica ring above the adsorbate. Similar adsorption patterns in the present experiment suggest that also Li penetrates the topmost oxide layer and binds to interfacial sites.

Both, the ring-shaped and $\mathrm{X}$-shaped Li elements are only observed at small sample bias but transform into round protrusions at elevated positive voltages (Fig. 2). The contrast change is accompanied by a large increase of the $\mathrm{Li}$ apparent height and occurs at a critical bias that depends on lateral size of the feature. Whereas an individual element turns bright at around $+2.75 \mathrm{~V}$, this value shifts to approximately $+2.25 \mathrm{~V}$ for aggregates containing more than three elements [as the one shown in Fig. 1(d)]. The apparent-height increase of Li indicates the availability of new conductance channels through the adsorbates at higher positive bias [Fig. 2(b)]. This assumption is corroborated by calculated state-density plots, which reveal a downshift of states in the silica film in 

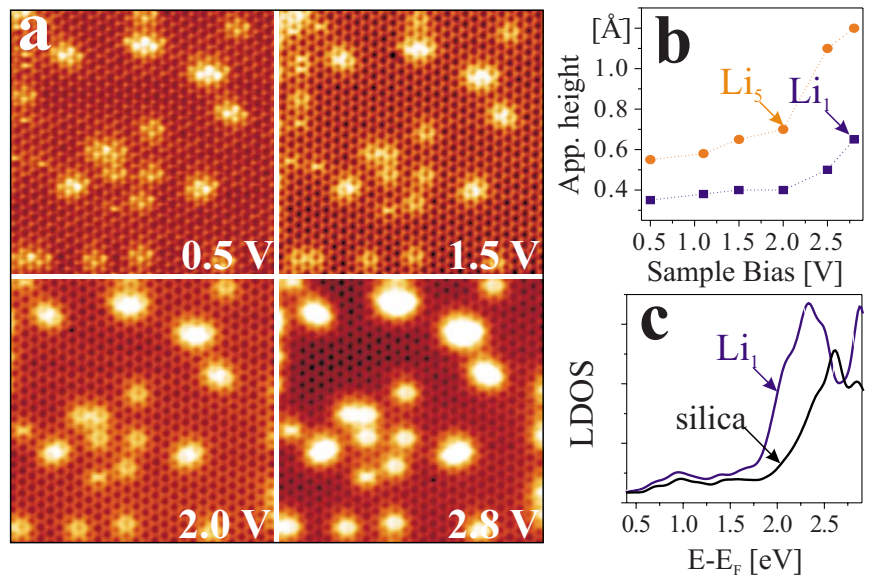

FIG. 2. (Color online) (a) Bias series of single Li atoms and small aggregates at the silica/Mo(112) interface $\left(15 \times 15 \mathrm{~nm}^{2}\right)$. (b) Evolution of the apparent Li height as a function of sample bias. (c) Calculated LDOS of the bare silica film and a single Li adsorbate. The experimental increase in $\mathrm{Li}$ apparent height is compatible with the higher state density above the embedded alkali species at higher energy.

vicinity to the Li atoms [Fig. 2(c)]. The origin of this electronic structure change is discussed in Sec. III C.

The Li adsorption behavior sketched above is in good agreement with DFT calculations on the silica/Mo(112) film. Single $\mathrm{Li}$ atoms are able to penetrate the openings in the -Si-O- hexagons with a small activation barrier of $0.3 \mathrm{eV}$. After passing the nanohole, the Li adsorbs in two different hollow sites along the $\operatorname{Mo}(112)$ furrows (Fig. 1). In the first site that is directly below a -Si-O- ring, the atom binds with $2.43 \mathrm{eV}$ and donates almost its complete $2 s$ electron to the Mo support (Bader charge: $+0.88|e|$ ). Additionally, the Li $2 s$ intermixes with the unoccupied $\mathrm{Si} 3 s-\mathrm{O} 2 p$ hybrid states in the silica ring above, thereby enhancing the empty-state density at the oxide surface [Fig. 2(c)]. This Li-induced increase of the local density of states (LDOS) is now probed in lowbias STM measurements and gives rise to the characteristic ringlike adsorption pattern shown in Fig. 1(a). Second, the Li can occupy a Mo hollow site below a [110]-oriented segment of a silica ring $\left(E_{\mathrm{B}}=2.41 \mathrm{eV}\right)$. In this configuration, the $\mathrm{Li} 2 s$ hybridizes with states in the $\mathrm{Si}-\mathrm{O}-\mathrm{Si}$ unit above, producing the X-shaped adsorption pattern of Fig. 1(b). Still, the $\mathrm{Li}$ interaction is dominated by the Mo states and involves the transfer of $0.82|e|$ out of the adsorbate according to a Bader analysis. The smaller net charge and the shorter Li-metal distance $(1.25 \AA$ versus $1.57 \AA$ ) with respect to the ring sites lead however to a $20 \%$ reduction in the induced surface dipole for $\mathrm{Li}$ in the bridge configuration.

In general, the adsorption characteristic of single $\mathrm{Li}$ atoms on the silica/Mo(112) film, as derived from STM and DFT data, follows essentially the well-established picture developed for metal supports. ${ }^{1,8}$ The Li becomes cationic in its interfacial binding configuration and occupies highcoordinated hollow sites along the Mo(112) furrows. The silica film above the Mo support seems to perturb this behavior only weakly. This situation changes however for higher Li loads as demonstrated in the next paragraph.

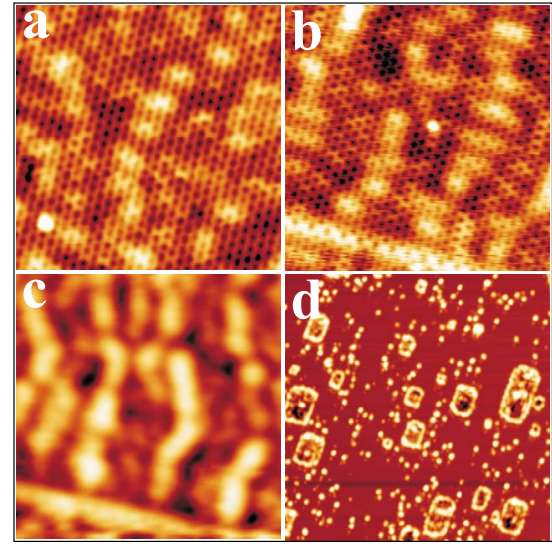

FIG. 3. (Color online) [(a)-(c)] STM topographic images of silica/Mo(112) taken for increasing Li exposures $\left(15 \times 15 \mathrm{~nm}^{2}\right)$. Bias voltages are $0.5 \mathrm{~V}$ in (a) and (b) and $2.5 \mathrm{~V}$ in (c). Atomic resolution is only obtained at low sample bias. The bright horizontal lines in (b) and (c) are Li-filled domain boundaries in the oxide film. (d) Sample surface at high Li load, when interfacial binding sites are filled and $\mathrm{Li}$ forms adclusters on the oxide surface $(3.5 \mathrm{~V}$, $150 \times 150 \mathrm{~nm}^{2}$ ).

\section{B. High Li coverage-Structural aspects}

\section{Results}

With increasing $\mathrm{Li}$ exposure, the characteristic ringshaped and X-shaped fingerprints of isolated Li atoms cannot be identified any more because the features overlap to complex adsorption patterns on the surface. However, Li-rich and Li-poor areas remain distinguishable due to an increased apparent height of the former in STM images taken above 2.0 V (Fig. 3). Surprisingly, the Li distribution at the metal-oxide interface becomes more and more inhomogeneous at higher alkali loads. Already at a nominal coverage of 0.2 atoms per pore, Li tends to assemble into groups of roughly half a dozen atoms sitting in adjacent pores [Fig. 3(a)]. This behavior amplifies with increasing exposure, when elongated $\mathrm{Li}$ islands start to develop at the silica-Mo interface. The aggregates adopt a certain short-range order with mean interisland distances of 30-50 $\AA$ and 15-20 $\AA$ along the [110] and [1111] directions of $\operatorname{Mo}(112)$, respectively [Figs. 3(a) and 3 (b)]. Above a critical coverage of $\Theta \sim 0.4$, long-range ordering effects become evident in the spatial distribution of the adsorbates. The Li-rich regions merge to [ $\overline{1} 11]$-oriented stripes of $15 \AA$ width and up to $200 \AA$ length, being delimited only by domain boundaries in the oxide film [Figs. 3(c) and 4(a)]. The islands roughly follow the orientation of the Mo(112) furrows, although local misalignments of up to $\pm 20^{\circ}$ are observed. The Li-rich stripes are separated by Lipoor regions, giving rise to a characteristic parallel-stripe pattern with $(44 \pm 4) \AA$ periodicity. This ordering phenomenon is not only observed in real space but leads to pronounced extra spots in Fourier-transformed images at a wave vector of $k_{110}^{-}=2 \pi / 45 \AA^{-1}$ [Fig. 4(b)]. An increase in the Li coverage above $\Theta \sim 0.6$ triggers the formation of a second set of [ $\overline{1} \overline{1} 1]$-oriented Li stripes, which are located in between the initial ones and appear with lower topographic contrast. 

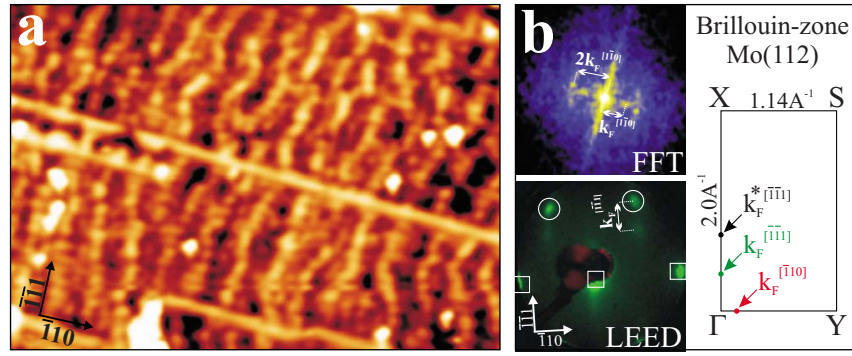

FIG. 4. (Color online) (a) Large scale STM image showing the ordered Li pattern on silica/Mo(112) $\left(3.0 \mathrm{~V}, 65 \times 45 \mathrm{~nm}^{2}\right)$. (b) Reciprocal space information deduced from a Fourier transformation of (a) and a LEED measurement performed at $30 \mathrm{eV}$ electron energy. The fundamental silica and Mo LEED spots are marked by circles and squares, respectively. The faint spot below each silica reflex reflects the long-range order in the Li distribution along the $\mathrm{Mo}[\overline{1} \overline{1} 1]$ direction. The [110] periodicity is not revealed due to the limited coherence length of the LEED system. The right inset depicts the Brillouin zone of $\mathrm{Mo}(112)$ with the $k$ vectors of prominent surface resonances marked by dots.

Consequently, the superstructure periodicity doubles to $(22 \pm 4) \AA$ in real space while a second reflex appears in the Fourier transformation [Fig. 4(b)].

Along the Li stripes, only weak and less regular intensity modulations are observed in the STM. However, low-energy electron-diffraction (LEED) measurements of the Li-doped silica film reveal an extra spot next to the silica reflex that indicates an additional periodicity of $(19 \pm 4) \AA$ along the $\mathrm{Mo}[\overline{1} \overline{1} 1]$ direction [Fig. 4(b)]. The $(44 \pm 4) \AA$ periodicity along the [110] direction, on the other hand, is not resolved in the diffraction pattern due to the limited coherence length of our LEED system.

It should be noted that also domain boundaries in the silica film appear with enhanced contrast in the STM, indicating a high concentration of interfacial $\mathrm{Li}$ ions (see, for instance, Fig. 3). This finding is not surprising, as the eightmembered rings along the boundaries are generally associated with reduced penetration barriers compared to the regular hexagons ${ }^{22,23}$ and should be easily filled with Li. For a nominal $\mathrm{Li}$ coverage exceeding one atom per pore, nucleation of Li adclusters is observed on the silica surface [Fig. 3(d)]. Apparently, all interfacial binding sites are saturated at this stage and atom penetration trough the oxide film is not feasible any more.

\section{Discussion}

The formation of spatially ordered $\mathrm{Li}$ islands at the silica-Mo interface deviates from the adsorption behavior observed on metal surfaces, where the alkali ions usually adopt open configurations in order to minimize their Coulomb repulsion. ${ }^{1,4,7}$ Even on the Mo(112) surface without oxide film, the development of $(4 \times 1)$ and $(2 \times 1)$ superstructures has been found in LEED upon alkali deposition. ${ }^{4,5}$ In contrast, the Li forms small assemblies at the silica-Mo interface already at relatively low exposure, although the calculated Bader charge of $+0.8|e|$ clearly indicates the ionic character of the $\mathrm{Li}$ species. The difference to bare metal surfaces can
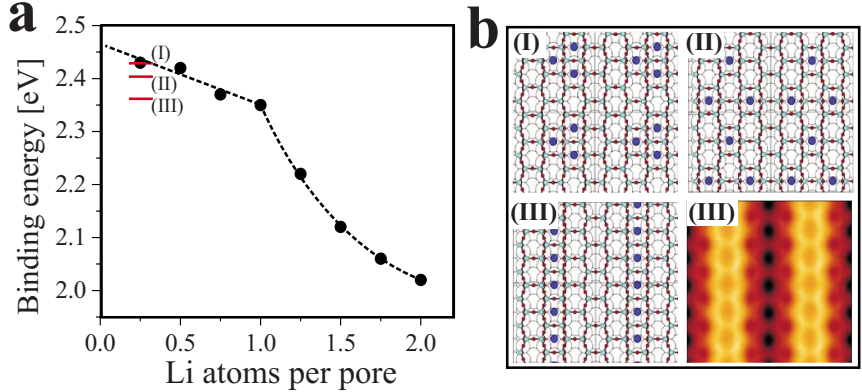

FIG. 5. (Color online) (a) Coverage dependence of the Li binding energy at the silica/Mo interface computed with a $(4 \times 2)$ supercell. (b) Structure model for three configurations with identical Li coverage of $\Theta=0.25$. The corresponding $\mathrm{Li}$ binding energies, being computed with a large $(6 \times 4)$ cell, are depicted in $(\mathrm{a})$. A simulated STM image for configuration (III) is shown in the lower right panel $\left(+2.0 \mathrm{~V}, 3.3 \times 3.3 \mathrm{~nm}^{2}\right)$. The Li-rich areas are imaged with enhanced contrast although the geometric corrugation is negligible, corroborating the strong electronic contribution to the STM contrast.

be related to the presence of the dielectric silica film on the Mo support, which contributes to the screening of the $\mathrm{Li}^{+}-\mathrm{Li}^{+}$ interaction. The screening efficiency becomes manifest in the weak coverage dependence of the Li binding energy, as computed with DFT [Fig. 5(a)]. An increase in the nominal coverage from zero to one $\mathrm{Li}^{+}$per silica pore reduces the adsorption strength by less than $3 \%$. Only a second Li inserted into the pores causes the binding energy to decline steeper due to the increasing Coulomb repulsion between the ions. This finding is in good agreement with the STM results, which indicate a maximum coverage of one $\mathrm{Li}$ per silica pore at the chosen experimental conditions. As incorporation of more $\mathrm{Li}$ would be favorable from a thermodynamic point of view, the saturation coverage seems to be related to a kinetic hindrance.

The weak initial coverage dependence of the $\mathrm{Li}$ binding energy enables the formation of $\mathrm{Li}$ islands at the metal-oxide interface. This effect can be illustrated by computing the stability of three hypothetical Li-silica structures with different interatomic spacing. In the first model, the $\mathrm{Li}$ ions are well separated while $\mathrm{Li}$ trimer and row structures are considered in the other two examples [Fig. 5(b)]. The trimer configuration turns out to be lowest in energy, whereas the isolated $\mathrm{Li}$ ions and the row pattern are less stable by only 0.025 and $0.07 \mathrm{eV}$ per ion, respectively. Although this energy difference is too small to conclude the thermodynamically favored $\mathrm{Li}$ distribution at the interface, the results clearly demonstrate that $\mathrm{Li}$ island formation is not hindered by the Coulomb repulsion between the $\mathrm{Li}^{+}$species in neighboring silica pores.

In order to analyze the driving force for the observed assembly effect, not only the interfacial binding energy but also the $\mathrm{Li}$ penetration process trough the silica top layer needs to be considered. Island formation would be favored if the Li penetration barrier is lowered in the presence of preadsorbed $\mathrm{Li}^{+}$at the interface, a mechanism that is indeed revealed in DFT. The initial penetration barrier for $\mathrm{Li}$ amounts to $\sim 0.3 \mathrm{eV}$ but gradually decreases when neighboring ad- 
sorption sites are occupied with alkali ions. In the limiting case of Li incorporation into a pore with six filled neighbors, the activation barrier drops to $0.11 \mathrm{eV}$ and atom penetration becomes comparably facile. The reduction in the barrier height hereby relates to a lattice distortion induced by the preadsorbed $\mathrm{Li}^{+}$ions at the interface.

The Li ordering process into [ $\overline{1} \overline{1} 1]$-oriented stripes on the silica-Mo(112) system cannot be treated with $a b$ initio DFT calculations, however already a simple analysis of the Mo(112) screening response provides valuable insight into the origin of pattern formation. Every adsorbate on a metal surface, especially if it holds an extra charge, perturbs the electron density of the support by initiating Friedel oscillations. ${ }^{24}$ Their periodicity is given by half the Fermi wavelength $\lambda_{\mathrm{F}}$ along a certain crystallographic direction while their amplitude decays with $k_{\mathrm{F}} r^{-2}$ as a function of distance $r$ from the binding site $\left(k_{\mathrm{F}}-\right.$ Fermi wave vector). Via this substrate-mediated interaction, adspecies are coupled over large distances on the metal surface, as they reciprocally influence the available charge density for binding. The perturbation is sufficiently strong to determine the spatial arrangement of adatoms and molecules on homogeneous surfaces, leading to distinct maxima in the pair-distribution function at multiples of $1 / 2 \lambda_{\mathrm{F}} \cdot{ }^{4,25-27}$

A similar mechanism can now be devised for the present adsorption system. Each $\mathrm{Li}$ ion creates a depletion zone in the $\mathrm{Mo}(112)$ charge density at $\sim 1 / 4 \lambda_{\mathrm{F}}$ around its adsorption site, whereas the electron density increases at $1 / 2 \lambda_{\mathrm{F}}$ and $\lambda_{\mathrm{F}}$. While adsorption of additional $\mathrm{Li}$ will be unfavorable within the depletion zone, it might be preferred in regions with higher electron density due to the superior screening ability of the substrate. The Friedel-type screening response is different along and perpendicular to the furrows of the Mo(112) surface [Fig. 4(b)]. According to bandstructure calculations ${ }^{28}$ and angle-resolved photoelectron spectroscopy, ${ }^{29}$ two surface resonances are of relevance along the $[\overline{1} 11]$-oriented furrows with $k_{\mathrm{F}}=0.36 \AA^{-1}\left(\lambda_{\mathrm{F}}\right.$ $=17.5 \AA)$ and $k_{\mathrm{F}}^{*}=0.86 \AA^{-1} \quad\left(\lambda_{\mathrm{F}}=7.3 \AA\right)$, respectively, whereas one band crosses the Fermi level at $k_{\mathrm{F}}=0.14 \AA^{-1}$ $\left(\lambda_{\mathrm{F}}=45 \AA\right)$ in $[\overline{1} 10]$ direction. $^{30}$ The latter periodicity matches the distance between neighboring Li stripes almost perfectly, suggesting that charge-density oscillations in the Mo surface are indeed responsible for the spatial distribution of the Li ions. Hereby, Li-rich stripes develop first with $\lambda_{\mathrm{F}}$ distance while the intermediated $1 / 2 \lambda_{\mathrm{F}}$ positions are only occupied at higher Li load. The retarded occupation of the half-wavelength sites indicates a residual influence of direct, repulsive interactions between adjacent $\mathrm{Li}$ islands. Using a similar argument, also the less pronounced Li-density modulations along the $[\overline{1} \overline{1} 1]$ stripes can be explained. STM images taken at low $\mathrm{Li}$ exposure and LEED data reveal a partly ordered Li distribution that is compatible with Friedel oscillations mediated by the surface resonance at $k_{\mathrm{F}}=0.36 \AA^{-1}$ [Figs. 3(c) and 4]. Its stabilizing effect on the $\mathrm{Li}^{+}$bindingenergy interferes however with direct interaction mechanisms, such as electrostatic forces between the $\mathrm{Li}^{+}$and the template effect of the silica/Mo film.

It should be noted that $\mathrm{Li}$ ordering into [ $\overline{1} \overline{1} 1]$ oriented, interfacial stripes is also supported by the strong anisotropy
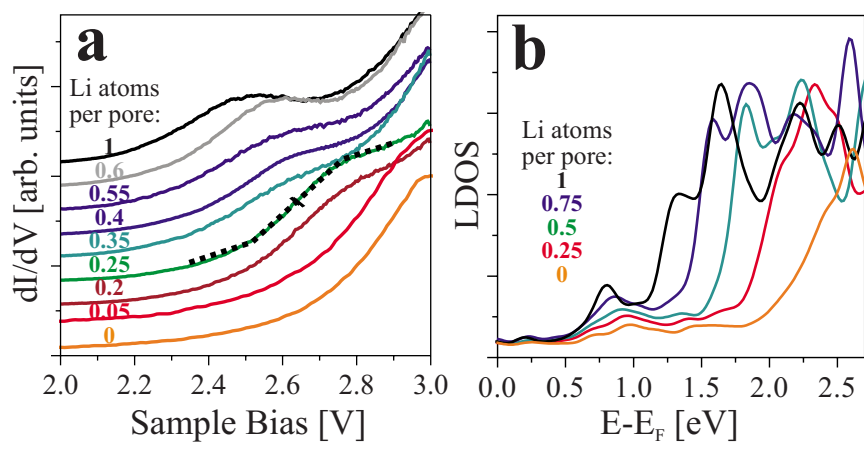

FIG. 6. (Color online) (a) Conductance spectra taken on silica/ $\mathrm{Mo}(112)$ regions with different $\mathrm{Li}$ concentrations. The set point for spectroscopy is adjusted to $2.9 \mathrm{~V}$; all spectra are offset for clarity. (b) Calculated silica/Mo LDOS as a function of Li concentration. The gradually downshift of the silica conductance states is clearly visible.

in the Mo(112) diffusion barriers. While Li diffusion perpendicular to the Mo furrows is activated by $0.75 \mathrm{eV}$, this value drops to $0.09 \mathrm{eV}$ along the $[\overline{1} 11]$ direction. ${ }^{31}$ The barrier rises to $0.27 \mathrm{eV}$ in presence of the silica film; however, transient $\mathrm{Li}$ diffusion remains possible along the furrows due to the thermal energy of the incoming atoms ( $800 \mathrm{~K}$ when leaving the Li dispenser). In contrast, hopping between the furrows is inhibited at the experimental conditions. Nonetheless, the succession of Li-rich and Li-poor islands can still be realized by inclining the stripes against the $\mathrm{Mo}[\overline{1} 11]$ direction, as revealed in the STM data.

\section{High Li coverage-Electronic aspects}

\section{Results}

The deposition of higher Li quantities strongly perturbs the electronic structure of the silica film, as demonstrated by conductance imaging and spectroscopy with the STM. A first indication comes from the large bias-dependent contrast between Li-rich and Li-poor surface regions. Similar to isolated Li species (Fig. 2), Li-rich islands exhibit large apparent heights of $1.2 \AA$ in images taken at elevated bias but are hardly visible at the Fermi level. The same effect is observed in simulated STM images [Fig. 5(b)], indicating an electronic origin of the measured surface corrugation. The fact that Li-rich stripes turn bright at higher bias values suggests the local availability of new conductance channels in those regions. STM conductance spectra taken on silica patches with different $\mathrm{Li}$ content provide direct evidence for this assumption [Fig. 6(a)]. The pristine film exhibits a smoothly increasing $d I / d V$ curve, interrupted only by a small kink at $\sim 3.0 \mathrm{~V}$. The kink marks the sudden availability of empty states for electron transport in the silica film. A similar step in the state density is revealed in the calculated LDOS [Fig. $6(b)]$ and assigned to the onset of unoccupied Si-O hybrid states, forming a precursor of the silica conduction band. Experimentally, the band onset $E_{c}$ is defined by the bias value, where either the slope of the $d I / d V$ curve becomes maximal or alternatively the $d I / d V$ signal reaches $50 \%$ of the kink intensity [see dashed line in Fig. 6(a)]. The so-estimated 


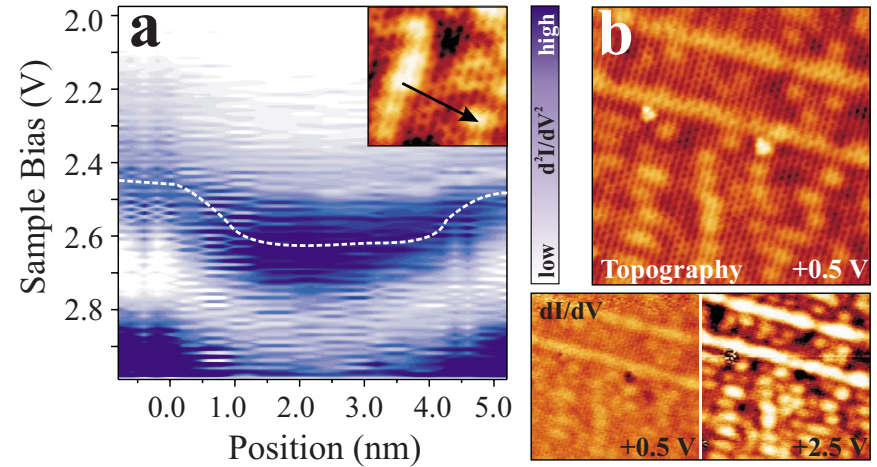

FIG. 7. (Color online) (a) Series of $64 d I / d V$ spectra taken along the line shown in the inset (STM: $0.5 \mathrm{~V}, 7 \times 7 \mathrm{~nm}^{2}$ ). Every spectrum has been differentiated with respect to the bias voltage and dark colors represent regions with large $d I / d V$ slopes. The dashed line approximates the position of maximum slope, which is taken as a measure for the conduction-band onset. (b) Topographic and conductance images of silica/Mo(112) loaded with nominally $0.3 \mathrm{Li}$ atoms per pore $\left(20 \times 20 \mathrm{~nm}^{2}\right)$. Whereas no $d I / d V$ contrast is visible at low bias, Li-rich regions appear bright in $d I / d V$ maps taken at $2.5 \mathrm{~V}$.

band onset at $2.9 \mathrm{~V}$, together with a similar kink at $-3.6 \mathrm{~V}$ in filled state spectra, provides an estimation of the band gap in the pristine silica film. Not surprisingly, it is much smaller than in bulk $\mathrm{SiO}_{2}\left(E_{\mathrm{g}} \sim 9 \mathrm{eV}\right),{ }^{32}$ reflecting the reduced oxide thickness and the residual influence of the metal support.

The kink in the empty-state spectra now experiences a gradual shift to lower energies with increasing Li coverage [Fig. 6(a)]. Additionally, it occurs at lower absolute $d I / d V$ intensity and considerably broadens with increasing $\mathrm{Li}$ load. ${ }^{33}$ A careful analysis of the experimental data indicates a monotonous downshift of the silica conduction states from 2.9 to $2.4 \mathrm{eV}$ as the $\mathrm{Li}$ coverage increases toward one atom per pore [Fig. 8(a)]. The band onset even moves to $\sim 2.3 \mathrm{eV}$ along the Li-filled domain boundaries.

A spatial correlation between the topographic and electronic properties of the Li-doped silica film is derived from spectral series taken across one period of the Li-induced superstructure. The $d I / d V$ spectra shown in Fig. 7(a) are recorded along the Mo[110] direction, differentiated with respect to the bias voltage and displayed as a color-coded matrix. In this representation, the position of maximum slope that marks the band onset $E_{c}$ is clearly visible due to its high $d^{2} I / d V^{2}$ signal. When moving the tip along the spectroscopy line, the band onset varies between 2.45 and $2.65 \mathrm{eV}$, indicating the inhomogeneous $\mathrm{Li}$ concentration at the interface. Whereas near-saturation coverage is revealed for the $\mathrm{Li}$ stripes, the interjacent regions have an $E_{c}$ value that is still well below the one for pristine film, suggesting a substantial $\mathrm{Li}$ concentration. Another way to convey the same information is $d I / d V$ mapping as a function of sample bias, as demonstrated in Fig. 7(b). In maps taken at $2.5 \mathrm{~V}$, the local downshift of the silica conduction band becomes evident from the high conductance signal above the Li-rich stripes while Lipoor regions remain dark in the image. At low sample bias $(0.5 \mathrm{~V})$, the $d I / d V$ contrast disappears, as the silica conduction band is not reached at any place of the surface.

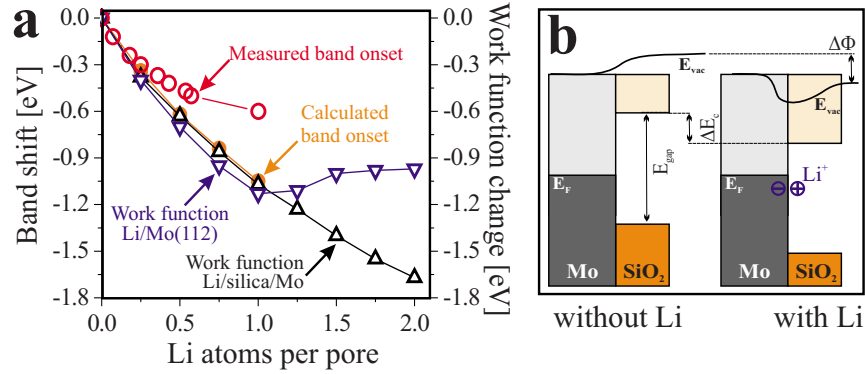

FIG. 8. (Color online) (a) Evolution of the measured and calculated conduction-state onsets and of the $\mathrm{SiO}_{2} / \mathrm{Mo}(112)$ and $\mathrm{Mo}(112)$ work function as a function of Li coverage. (b) Sketch of the silica/Mo electronic structure before and after Li incorporation. The model visualizes the interrelation between the Li-induced surface dipole, the work function change $\Delta \Phi$ and the downshift of the silica conduction states $\Delta E_{c}$.

\section{Discussion}

The gradual downshift of silica conduction states with increasing $\mathrm{Li}$ load can be rationalized by a work-function reduction in silica/Mo(112) upon $\mathrm{Li}$ incorporation, in agreement with the old Langmuir-Gurney model. ${ }^{8}$ The positive surface dipole formed by interfacial $\mathrm{Li}^{+}$and image charges in the metal triggers a lowering of the vacuum energy of the system. As the oxide electronic states are mainly adjusted to the vacuum level, the band positions experience an equivalent downshift with increasing Li load [Fig. 8(b)]. The direct proportionality between work function and band onsets in this case is related to the large gap value of silica, which prevents any charge transfer into the oxide film and hence a pinning of the energy levels. The dependence of work function $\phi$ and silica band onsets $E_{c}$ on the Li coverage has been calculated by DFT and is depicted in Fig. 8(a). For low Li concentrations, the work function decreases almost linearly with coverage according to $\delta \phi / \delta \Theta=-1.3 \mathrm{eV}$ per Li-filled pore. The slope gradually decreases for higher coverage but remains negative also for two $\mathrm{Li}^{+}$per silica ring $(\delta \phi / \delta \Theta=$ $-0.65 \mathrm{eV} / \mathrm{Li})$. Even at this maximum load, the work function of the system is clearly higher than the tabulated Li-bulk value of $2.9 \mathrm{eV}$ (Ref. 34) [which would correspond to $\Delta \phi$ $=-2.0 \mathrm{eV}$ in Fig. 8(a)]. This finding suggests that the charge transfer out of the Li does not saturate and the alkali atoms remain cationic at the silica-Mo interface. Apparently, metallization of the alkali layer is prevented by the small number of atoms that fit into the silica-Mo interface region. Bulk values for the Li work function are only expected if all interfacial sites are saturated and Li starts nucleating above the oxide surface. Although this cluster formation is observed experimentally [Fig. 3(d)], the associated work function is not accessible to STM any more due to the vanishing influence on the silica conduction states in this case.

In contrast to the $\mathrm{Li} /$ silica/Mo case, the universal work function versus coverage curve is restored on the bare Mo(112) surface [Fig. 8(a)]. There, $\phi$ experiences an initial decline with Li coverage but runs through a minimum at $\Theta$ $\sim 1$ and increases again toward the Li-bulk value. The different behavior of the oxide-covered system is traced back to the special role of the interfacial $\mathrm{O}$ atoms in the silica film, 
which act as an additional sink for the Li electrons and further screen the Li charge distribution at the interface. Not surprisingly, the universal coverage dependence of $\phi$ also reappears when the alkali atoms do not bind at the interface but on top of the oxide film where screening is inefficient. Such a behavior is revealed, for instance, for $\mathrm{K}$ atoms, which are too big to penetrate the silica nanopores. ${ }^{11}$

The experimentally determined onset of the silica conduction states mimics the computed trend with Li coverage, although absolute values are $40 \%$ smaller [Fig. 8(a)]. This discrepancy can be explained by the known deficiency of DFT in reproducing the correct band gaps of insulating materials. On the experimental side, the Li-induced band shift might partly be compensated by the tip-induced counter field during spectroscopy. Nonetheless, experimental and theoretical data consistently demonstrate the strong effect of incorporated $\mathrm{Li}^{+}$on the silica/Mo work function.

\section{CONCLUSIONS}

The adsorption behavior of $\mathrm{Li}$ on a silica thin-film grown on Mo(112) has been characterized by low-temperature STM and DFT calculations. Due to the porous structure of the silica, $\mathrm{Li}$ atoms are able to penetrate the oxide layer and bind in two distinct configurations at the metal-oxide interface. Substrate-mediated interactions between the Li ions give rise to their organization into elongated Li stripes at higher alkali exposure. Upon adsorption, the Li becomes cationic and forms a positive surface dipole, which in turn reduces the work function of the silica/Mo(112) film. This work function decrease leads to a downshift of the silica conduction band, reaching $0.5 \mathrm{eV}$ (theoretical value $1.0 \mathrm{eV}$ ) when one $\mathrm{Li}$ ion occupies each silica pore.

The Li-induced modification of the silica/Mo(112) electronic properties and, in particular, the possibility to tune its work function opens various interesting applications. In contrast to the pristine film, Li-doped silica is attractive for the adsorption of electronegative species, as the reduced work function promotes charge-transfer processes out of the film. ${ }^{12}$ By this means, anionic species can be stabilized on the oxide surface, being a promising starting point to fabricate systems with enhanced chemical activity. With respect to other techniques, the modification of the oxide properties is hereby based on a "passive" approach, as the Li ions are neither directly involved nor consumed in the adsorption process. The tuning of electronic and chemical properties of oxide films via alkali doping has universal character and can, in principle, be applied to other systems. Although Li insertion into silica/Mo(112) is particularly easy due to the porous nature of the film, the technique can be extended to compact oxide materials, where the alkali atoms need to be introduced prior to film growth.

\section{ACKNOWLEDGMENTS}

The work has been supported by the COST Action D41. Part of the computing time was provided by the Barcelona Supercomputing Center (BSC-CNS).
*Corresponding author; nilius@ fhi-berlin.mpg.de

${ }^{1}$ H. P. Bonzel, Surf. Sci. Rep. 8, 43 (1988).

${ }^{2}$ R. D. Diehl and R. McGrath, Surf. Sci. Rep. 23, 43 (1996).

${ }^{3}$ D. M. Riffe, G. K. Wertheim, and P. H. Citrin, Phys. Rev. Lett. 64, 571 (1990); S. R. Chubb, E. Wimmer, A. J. Freeman, J. R. Hiskes, and A. M. Karo, Phys. Rev. B 36, 4112 (1987).

${ }^{4}$ I. N. Yakovkin, Surf. Sci. 282, 195 (1993); 559, 29 (2004); J. Nanosci. Nanotechnol. 1, 357 (2001).

${ }^{5}$ A. Fedorus, D. Kolthoff, V. Koval, I. Lyuksyutov, A. G. Naumovets, and H. Pfnür, Phys. Rev. B 62, 2852 (2000).

${ }^{6}$ G. Doyen, D. Drakova, J. V. Barth, R. Schuster, T. Gritsch, R. J. Behm, and G. Ertl, Phys. Rev. B 48, 1738 (1993).

${ }^{7}$ J. Kliewer and R. Berndt, Surf. Sci. 477, 250 (2001).

${ }^{8}$ I. Langmuir and K. H. Kingdon, Science 57, 58 (1923); I. Langmuir, J. Am. Chem. Soc. 54, 2798 (1932); R. W. Gurney, Phys. Rev. 47, 479 (1935).

${ }^{9}$ Handbook of Heterogeneous Catalysis, 2th ed., edited by G. Ertl, H. Knoezinger, F. Schueth, and J. Weitkamp (Wiley-VCH, Weinheim, 2008).

${ }^{10}$ J. H. Lunsford, Angew. Chem., Int. Ed. 34, 970 (1995).

${ }^{11}$ U. Martinez, L. Giordano, and G. Pacchioni, J. Chem. Phys. 128, 164707 (2008).

${ }^{12}$ U. Martinez, J.-F. Jerratsch, N. Nilius, L. Giordano, G. Pacchioni, and H.-J. Freund, Phys. Rev. Lett. 103, 056801 (2009).

${ }^{13}$ G. Pacchioni, L. Giordano, and M. Baistrocchi, Phys. Rev. Lett. 94, 226104 (2005).
${ }^{14}$ J. Weissenrieder, S. Kaya, J. L. Lu, H. J. Gao, S. Shaikhutdinov, H.-J. Freund, M. Sierka, T. K. Todorova, and J. Sauer, Phys. Rev. Lett. 95, 076103 (2005).

${ }^{15}$ L. Giordano, D. Ricci, G. Pacchioni, and P. Ugliengo, Surf. Sci. 584, 225 (2005).

${ }^{16}$ J. B. Giorgi, T. Schröder, M. Bäumer, and H.-J. Freund, Surf. Sci. Lett. 498, L71 (2002).

${ }^{17}$ T. K. Todorova, M. Sierka, J. Sauer, S. Kaya, J. Weissenrieder, J.-L. Lu, H.-J. Gao, S. K. Shaikhutdinov, and H.-J. Freund, Phys. Rev. B 73, 165414 (2006).

${ }^{18}$ G. Kresse and J. Hafner, Phys. Rev. B 47, 558 (1993); G. Kresse and J. Furthmüller, ibid. 54, 11169 (1996).

${ }^{19}$ J. P. Perdew, J. A. Chevary, S. H. Vosko, K. A. Jackson, M. R. Pederson, D. J. Singh, and C. Fiolhais, Phys. Rev. B 46, 6671 (1992).

${ }^{20}$ P. E. Blöchl, Phys. Rev. B 50, 17953 (1994).

${ }^{21}$ J. Tersoff and D. R. Hamann, Phys. Rev. Lett. 50, 1998 (1983).

${ }^{22}$ S. Ulrich, N. Nilius, H.-J. Freund, L. Giordano, and G. Pacchioni, ChemPhysChem 9, 1367 (2008).

${ }^{23}$ S. Ulrich, N. Nilius, H.-J. Freund, U. Martinez, L. Giordano, and G. Pacchioni, Surf. Sci. 603, 1145 (2009).

${ }^{24}$ T. Einstein, Physical Structure of Solid Surface, edited by W. N. Unertl (North-Holland, Amsterdam/Elsevier, New York, 1996).

${ }^{25}$ R. Casanova and T. T. Tsong, Phys. Rev. B 22, 5590 (1980); T. T. Tsong and R. Casanova, ibid. 24, 3063 (1981).

${ }^{26}$ V. Simic-Milosevic, M. Heyde, N. Nilius, M. Nowicki, H.-P. 
Rust, and H.-J. Freund, Phys. Rev. B 75, 195416 (2007).

${ }^{27}$ J. Repp, F. Moresco, G. Meyer, K.-H. Rieder, P. Hyldgaard, and M. Persson, Phys. Rev. Lett. 85, 2981 (2000).

${ }^{28}$ I. N. Yakovkin, Surf. Sci. 389, 48 (1997).

${ }^{29}$ J. Zhang, D. N. McIlroy, and P. A. Dowben, Phys. Rev. B 49, 13780 (1994).

${ }^{30}$ The states are surface resonances with a strong bulk contribution and not genuine surface states and therefore less susceptible to the presence of the silica film above. However, small shifts of the wave vectors can be expected on the oxide covered with respect to the bare $\mathrm{Mo}(112)$ surface.

${ }^{31}$ A. Kiejna and R. M. Nieminen, Phys. Rev. B 66, 085407 (2002).

${ }^{32}$ N. F. Mott, J. Non-Cryst. Solids 40, 1 (1980).

${ }^{33}$ The spectral broadening reflects a certain averaging over oxide regions with different Li content.

${ }^{34}$ J. Hoelz and F. K. Schulte, Work Function of Metals (Springer, Berlin, 1979). 